Check for updates

Cite this: Phys. Chem. Chem. Phys., 2020, 22, 9703

Received 19th February 2020 Accepted 18th March 2020

DOI: $10.1039 / \mathrm{d} 0 \mathrm{cp} 00935 \mathrm{k}$

rsc.li/pccp

\title{
Generalised magnetisation-to-singlet-order transfer in nuclear magnetic resonance $\dagger$
}

\author{
Christian Bengs, (D) a Mohamed Sabba, ${ }^{a}$ Alexej Jerschow ${ }^{\mathrm{b}}$ and \\ Malcolm H. Levitt iD *a
}

\begin{abstract}
A variety of pulse sequences have been described for converting nuclear spin magnetisation into longlived singlet order for nuclear spin-1/2 pairs. Existing sequences operate well in two extreme parameter regimes. The magnetisation-to-singlet (M2S) pulse sequence performs a robust conversion of nuclear spin magnetisation into singlet order in the near-equivalent limit, meaning that the difference in chemical shift frequencies of the two spins is much smaller than the spin-spin coupling. Other pulse sequences operate in the strong-inequivalence regime, where the shift difference is much larger than the spin-spin coupling. However both sets of pulse sequences fail in the intermediate regime, where the chemical shift difference and the spin-spin coupling are roughly equal in magnitude. We describe a generalised version of M2S, called gM2S, which achieves robust singlet order excitation for spin systems ranging from the near-equivalence limit well into the intermediate regime. This closes an important gap left by existing pulse sequences. The efficiency of the gM2S sequence is demonstrated numerically and experimentally for near-equivalent and intermediate-regime cases.
\end{abstract}

\section{Introduction}

Nuclear long-lived spin order refers to spin ensemble configurations with exceptional relaxation time constants. Such configurations are protected against many important relaxation mechanisms and may exhibit life times that greatly exceed the longitudinal spin-lattice relaxation time $\sim T_{1} \cdot{ }^{1-5}$ In certain cases nuclear long-lived spin order may persist for tens of minutes. ${ }^{6-9}$

The long-lived behaviour of such spin configurations has been integrated into a multitude of experimental protocols. ${ }^{10-12}$ In the context of diffusion NMR long-lived spin states have enabled the study of previously inaccessible spatial and dynamical regimes. ${ }^{13-17}$ For the field of hyperpolarisation NMR long-lived spin modes represent promising candidates for the "storage" of an enhanced magnetic response, and its readout at convenient times. ${ }^{18-28}$ More recently, techniques of this type have been applied to the study of bio-molecular markers and their intricate interactions with their surroundings. ${ }^{29-34}$

Many experiments in singlet-assisted NMR exploit nearequivalent spin-1/2 pairs, meaning that the difference in chemically shifted resonance frequencies for the two spins $\Delta$

\footnotetext{
${ }^{a}$ School of Chemistry, University of Southampton, University Road, SO17 1BJ, UK. E-mail:mhl@soton.ac.uk

${ }^{b}$ Department of Chemistry, New York University, New York, NY 10003, USA.

E-mail: alexej.jerschow@nyu.edu

$\dagger$ Electronic supplementary information (ESI) available. See DOI: 10.1039/ d0cp00935k
}

is much smaller than the scalar coupling constant $J$ (both $\Delta$ and $J$ are defined in $\mathrm{Hz}$ ). In the near-equivalent regime, the eigenstates of the spin Hamiltonian are close to the singlet and triplet states, defined as follows

$$
\begin{gathered}
\left|S_{0}\right\rangle=(|\alpha \beta\rangle-|\beta \alpha\rangle) / \sqrt{2}, \quad\left|T_{+1}\right\rangle=|\alpha \alpha\rangle, \\
\left|T_{0}\right\rangle=(|\alpha \beta\rangle+|\beta \alpha\rangle) / \sqrt{2}, \quad\left|T_{-1}\right\rangle=|\beta \beta\rangle .
\end{gathered}
$$

Singlet spin order (SO) is defined as the difference between the singlet population and the mean triplet population

$$
\mathrm{SO}=\left|S_{0}\right\rangle\left\langle S_{0}\left|-\frac{1}{3} \sum_{m=-1}^{+1}\right| T_{m}\right\rangle\left\langle T_{m}\right| .
$$

In near-equivalent spin-pairs, defined by the condition $|\Delta| \ll|J|$, singlet order often exhibits a long lifetime without any further intervention, due to strong correlations in the fluctuating magnetic fields responsible for relaxation. ${ }^{35}$ In the intermediate coupling regime $(|\Delta| \sim|J|)$, or the strong inequivalence regime $(|\Delta| \gg|J|)$, on the other hand, singlet order only reveals its long-lived nature when it is "locked" or "sustained" by applying resonant radio-frequency fields. ${ }^{2,5,36}$ To a good approximation, the strong resonant radio-frequency field imposes magnetic equivalence on the effective spin Hamiltonian, so that the Hamiltonian eigenstates in the presence of the field are given, to a good approximation, by the singlet and triplet states defined in eqn (1). 
In this article we quantify the inequivalence of the spin system by the singlet-triplet mixing angle $\theta_{\mathrm{ST}}$, defined as follows

$$
\theta_{\mathrm{ST}}=\tan ^{-1}\left(\frac{\Delta}{J}\right)
$$

Near-equivalent systems have $\theta_{\mathrm{ST}} \approx 0$. Strongly inequivalent systems (weakly coupled spin pairs) have $\theta_{\mathrm{ST}} \approx \pi / 2$. The intermediate coupling regime is defined by $\theta_{\mathrm{ST}} \approx \pi / 4$.

Singlet order is usually accessed by applying a radio-frequency pulse sequence which converts nuclear magnetisation along the field (represented by the operator $I_{z}$ ) into the singlet order operator of eqn (2). Several radio-frequency (rf) pulse sequences have been developed for this purpose..$^{2,6,36-42}$ However, most pulse sequences are designed for either the near-equivalent regime $\left(\theta_{\mathrm{ST}} \approx 0\right)$, or the strongly inequivalent regime $\left(\theta_{\mathrm{ST}} \approx \pi / 2\right)$. The intermediate coupling regime $\left(\theta_{\mathrm{ST}} \approx \pi / 4\right)$ is a difficult case which is not welladdressed by most existing sequences.

The normalised amplitude for the conversion of Zeeman order into singlet order is defined here as follows

$$
\zeta=\left(Q_{\mathrm{so}} \mid \hat{U} Q_{z}\right)
$$

where the normalised operators for $z$-polarisation and singlet order are given by

$$
\begin{aligned}
Q_{z} & =\frac{1}{\sqrt{2}} I_{z} \\
Q_{\mathrm{SO}} & =-\frac{2}{\sqrt{3}} \mathbf{I}_{\mathbf{1}} \cdot \mathbf{I}_{\mathbf{2}}=\frac{\sqrt{3}}{2} \mathrm{SO} .
\end{aligned}
$$

Here $\hat{U}$ is the propagation superoperator for the spin dynamics under the pulse sequence, ${ }^{43}(A \mid B)=\operatorname{Tr}\left\{A^{\dagger} B\right\}$ is the Liouville bracket, ${ }^{43}$ and the operators $Q_{z}$ and $Q_{\text {so }}$ are normalised such that $\left(Q_{z} \mid Q_{z}\right)=\left(Q_{\text {so }} \mid Q_{\text {so }}\right)=1$. The operator $Q_{\text {so }}$ is a normalised version of the singlet order operator SO in eqn (2). For the case of unitary transformations, as generated by coherent radio-frequency pulse sequences without relaxation, the transformation amplitude in eqn (4) is subject to strict bounds which may be derived from the eigenvalue spectra of the relevant operators. ${ }^{44}$ In the current case, the relevant unitary bound is as follows ${ }^{44,45}$

$$
|\zeta| \leq \zeta_{\max }
$$

where

$$
\zeta_{\max }=\sqrt{2 / 3}
$$

This means that in the absence of relaxation, no pulse sequence can convert more than $\sqrt{2 / 3} \simeq 81.6 \%$ of the normalised Zeeman order $Q_{z}$ into normalised singlet order $Q_{\text {so. }}$. In most cases, relaxation leads to further losses.

The magnetisation-to-singlet conversion amplitudes $\zeta$ are plotted against the singlet-triplet mixing angles for several different pulse sequences in Fig. 1.

The magnetisation-to-singlet conversion amplitude of the M2S sequence ${ }^{37,38}$ is shown by the orange line. This simulation uses the optimum values for the M2S pulse sequence parameters given in Table 1 and described below.

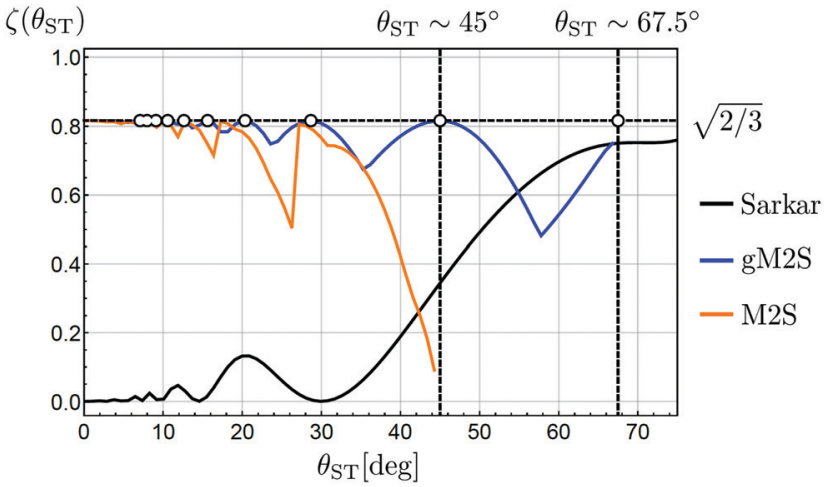

Fig. 1 Transformation amplitudes $\zeta$ for Zeeman polarisation into singlet order (eqn (4)), as a function of mixing angle $\theta_{\mathrm{ST}}$. The near-equivalent regime $\left(\theta_{\mathrm{ST}} \approx 0\right)$ is shown on the left of the plot, with the strong inequivalence (weak coupling) regime $\left(\theta_{\mathrm{ST}} \approx \pi / 2\right)$ on the right, and the intermediate regime $\left(\theta_{\mathrm{ST}} \approx \pi / 4\right)$ in the middle. The transformation amplitudes $\zeta$ of the M2S, ${ }^{37}$ Sarkar $^{46}$ and gM2S pulse sequences, are plotted. Relaxation is ignored in all cases. The pulse sequence parameters for the Sarkar sequence uses the analytic solutions in ref. 46 . The pulse sequence parameters for the M2S and gM2S sequences use the analytic solutions in Table 1. Circles indicate the mixing angles $\theta_{\mathrm{ST}}$ for which the gM2S sequence provides optimal efficiency $\left(\zeta=\zeta_{\text {max }}\right)$.

The performance of M2S reaches the theoretical limit of $\zeta=\zeta_{\max }$ in the near-equivalence regime (small values of $\theta_{\mathrm{ST}}$ ). However the performance of M2S starts to oscillates when $\theta_{\mathrm{ST}}$ exceeds $\sim 20^{\circ}$ and collapses completely for $\theta_{\mathrm{ST}} \gtrsim 40^{\circ}$. Other sequences for the near-equivalence regime, such as SLIC (spinlock-induced crossing), ${ }^{40}$ also fail outside the near-equivalence regime.

The pulse sequence proposed by Sarkar et al., ${ }^{46}$ on the other hand, has a performance shown by the black curve in Fig. 1. This sequence achieves near-optimal magnetisation-to-singlet conversion for $\theta_{\mathrm{ST}} \gtrsim 60^{\circ}$ (strong inequivalence) but its performance declines steeply below $\theta_{\text {ST }} \lesssim 55^{\circ}$. Other proposed sequences for the strong-inequivalence regime ${ }^{2,6}$ have similar behaviour.

There have been several proposals for filling in the lacuna around $\theta_{\mathrm{ST}} \approx 45^{\circ}$.

One approach is to introduce multiple-pulse chemical-shift scaling (CSS) into the magnetisation-to-singlet (M2S) pulse sequence. ${ }^{47}$ The resulting method is rather complex and involves the application of a large number of pulses. The sequence is prone to error accumulation and may give rise to sample heating. ${ }^{48}$

An alternative method is the homonuclear ADAPT (Alternating Delays Achieve Polarization Transfer) technique. ${ }^{49,50}$ This sequence consists of a repetitive sequence of short pulses. Simulations show that in ideal circumstances this sequence performs well for mixing angles given by $0<\theta_{\mathrm{ST}} \lesssim 75^{\circ}$. However, ADAPT is not a robust method. As mentioned in ref. 50, the ADAPT sequence suffers from strong interference from off-resonance effects and radio-frequency field inhomogeneity. The performance of ADAPT is explored in more detail in the ESI. $\dagger$

A different approach is to apply radio-frequency fields with computer-optimised variations of amplitude and phase to induce the required transformations. This includes the use of optimal control theory, ${ }^{51}$ and the set of techniques called 
Table 1 Spin system parameters, optimal delays $\tau_{1}^{*}, \tau_{2}^{*}$, and optimal echo number $n^{\star}$ for the M2S and gM2S sequences

\begin{tabular}{lll}
\hline & M2S & gM2S \\
\hline$\theta_{\mathrm{ST}}$ & $\tan ^{-1}(\Delta / J)$ & $\tan ^{-1}(\Delta / J)$ \\
$\omega_{\mathrm{e}}$ & $2 \pi \sqrt{J^{2}+\Delta^{2}}$ & $2 \pi \sqrt{J^{2}+\Delta^{2}}$ \\
$\xi^{12}$ & $2 \sin ^{-1}(\Delta / J)$ & $2 \sin ^{-1}(\sqrt{2} \Delta /(J+\Delta))$ \\
$n^{*}$ & $\operatorname{round}\left(\pi /\left(2 \xi^{12}\right)\right)$ & $\operatorname{round}\left(\pi / \xi^{12}\right)$ \\
$\tau_{1}^{*}$ & $2 \omega_{\mathrm{e}}^{-1} \tan ^{-1}\left(\sqrt{\left(J^{2}+\Delta^{2}\right) /\left(J^{2}-\Delta^{2}\right)}\right)$ & $\omega_{\mathrm{e}}^{-1} \cos ^{-1}(\Delta(J-\Delta) /(J(J+\Delta)))$ \\
$\tau_{2}^{*}$ & $\omega_{\mathrm{e}}^{-1} \tan ^{-1}\left(\cos \left(\theta_{\mathrm{ST}}\right) \cot \left(2 n^{*} \tau_{1}^{*} \omega_{\mathrm{e}} \cos \left(\theta_{\mathrm{ST}}\right)\right)\right)$ & $\omega_{\mathrm{e}}^{-1}\left(\left|\tan ^{-1}\left(\cot \left(2 n^{*} \tau_{1}^{*} \omega_{\mathrm{e}} \cos \left(\theta_{\mathrm{ST}}\right)\right) \sec \left(\theta_{\mathrm{ST}}\right)\right)\right|+2 \pi\right) \quad$ for $n^{*}=1$ \\
& & $\omega_{\mathrm{e}}^{-1}\left|\tan ^{-1}\left(\cot \left(2 n^{*} \tau_{1}^{*} \omega_{\mathrm{e}} \cos \left(\theta_{\mathrm{ST}}\right)\right) \sec \left(\theta_{\mathrm{ST}}\right)\right)\right| \quad$ for $n^{*}>1$
\end{tabular}

APSOC (adiabatic passage spin-order conversion). ${ }^{41,42}$ Although such techniques are often efficient and robust, they have the disadvantage that there are no analytical solutions; in many cases, specific shapes must be derived for each set of spin system parameters. We do not consider these schemes further in this paper.

In this work we present a generalised M2S sequence (gM2S) which provides robust magnetisation-to-singlet transfer efficiency for systems ranging from near-equivalence well into the intermediate regime $\left(0<\theta_{\mathrm{ST}} \lesssim 67.5^{\circ}\right)$. The parameters of the gM2S sequence are described by analytical equations for the case of infinitely short rf pulses. The performance of gM2S is shown by the blue line in Fig. 1. It covers the gap in performance between existing pulse sequences rather well, and, as discussed below, its performance is very robust with respect to common experimental imperfections.

\section{Theory}

\subsection{Singlet-triplet evolution}

The rotating-frame Hamiltonian for a coupled two-spin-1/2 system in solution may be expressed as follows: ${ }^{52}$

$$
\begin{aligned}
H_{0} & =\frac{1}{2} \omega_{\Delta}\left(I_{1 z}-I_{2 z}\right)+\omega_{J} I_{1} \cdot I_{2}, \\
\omega_{J} & =2 \pi J, \quad \omega_{\Delta}=\omega_{1}^{0}-\omega_{2}^{0}=2 \pi \Delta .
\end{aligned}
$$

The reference frequency is centred between the resonance frequencies $\omega_{j}^{0}$ of the two spins, so that $\omega_{\Delta}$ describes their (rotating-frame) resonance frequency difference and $\omega_{J}$ the mutual scalar coupling. The eigenstates of the Hamiltonian for the case that $\omega_{\Delta}=0$ are given by the singlet and triplet states in eqn (1).

This suggests the following re-parametrisation of the Hamiltonian

$$
H_{0}=\omega_{\mathrm{e}}\left(\frac{1}{2} \sin \left(\theta_{\mathrm{ST}}\right)\left(I_{1 z}-I_{2 z}\right)+\cos \left(\theta_{\mathrm{ST}}\right)\left(I_{1} \cdot I_{2}+\frac{1}{4} 1\right)\right),
$$

with

$$
\omega_{\mathrm{e}}=2 \pi \sqrt{J^{2}+\Delta^{2}}
$$

Eqn (9) shows that the mixing angle $\theta_{\mathrm{ST}}$ is the important physical quantity and the effective frequency $\omega_{\mathrm{e}}$ simply rescales the time axis.

Define a basis $\mathscr{B}$ spanned by the following basis states

$$
\mathscr{B}=\left(\begin{array}{c}
|1\rangle \\
|2\rangle \\
|3\rangle \\
|4\rangle
\end{array}\right)=\left(\begin{array}{c}
\left|S_{0}\right\rangle \\
\left|T_{0}\right\rangle \\
\frac{1}{\sqrt{2}}\left(\left|T_{+1}\right\rangle+\left|T_{-1}\right\rangle\right) \\
\frac{1}{\sqrt{2}}\left(\left|T_{+1}\right\rangle-\left|T_{-1}\right\rangle\right)
\end{array}\right),
$$

where the singlet and triplet states are defined in eqn (1). The Hamiltonian of eqn (8) decouples into two orthogonal subspaces

$$
\begin{aligned}
H_{0} & =H_{0}^{12}+H_{0}^{34} \\
& =\omega_{\mathrm{e}}\left(\left\{\sin \left(\theta_{\mathrm{ST}}\right) I_{x}^{12}-\cos \left(\theta_{\mathrm{ST}}\right) I_{z}^{12}\right\}+\left\{\frac{1}{2} \cos \left(\theta_{\mathrm{ST}}\right) 1^{34}\right\}\right),
\end{aligned}
$$

where the operators $I_{\mu}^{r s}$ represent single-transition operators along the Cartesian axis $\mu .^{53,54}$ The free evolution propagator of the system $U_{0}(\tau)$ may be written as follows:

$$
\begin{aligned}
& U_{0}(\tau)=\Phi^{34}\left(\frac{1}{2} \tau \cos \left(\theta_{\mathrm{ST}}\right) \omega_{\mathrm{e}}\right) \exp \left\{-i \tau \boldsymbol{\omega}^{12} \cdot \mathbf{I}^{12}\right\}, \\
& \boldsymbol{\omega}^{12}=\omega_{\mathrm{e}}\left[\sin \left(\theta_{\mathrm{ST}}\right) \quad 0 \quad-\cos \left(\theta_{\mathrm{ST}}\right)\right], \\
& \mathbf{I}^{r s}=\left[\begin{array}{lll}
I_{x}^{r s} & I_{y}^{r s} & I_{z}^{r s}
\end{array}\right] .
\end{aligned}
$$

where the propagator $\Phi^{r s}(\gamma)$ describes pure phase evolution in the subspace $\{|r\rangle,|s\rangle\}$ through the angle $\gamma$ :

$$
\Phi^{r s}(\gamma)=\exp \left\{-i \gamma \mathbf{1}^{r s}\right\}
$$

\subsection{Spin echoes}

The sequences described below make extensive use of spinecho (SE) blocks, of the form $\tau-180_{y}-\tau$, where $\tau$ denotes the duration of a delay interval. The propagator for a spin echo block is given by 


$$
U_{\mathrm{SE}}(\tau)=U_{0}(\tau) R_{y}(\pi) U_{0}(\tau)
$$

The rotation operators $R_{x}(\pi / 2), R_{y}(\pi / 2)$ and $R_{y}(\pi)$ may be expressed in terms of single-transition rotation operators as follows:

$$
\begin{aligned}
R_{x}(\pi / 2) & =R_{x}^{23}(\pi), \\
R_{y}(\pi / 2) & =R_{y}^{24}(-\pi), \\
R_{y}(\pi) & =i R_{z}^{12}(\pi) R_{z}^{34}(\pi) .
\end{aligned}
$$

The total echo propagator $U_{\mathrm{SE}}(\tau)$ may be written as the product of independent propagators in the $\{|1\rangle,|2\rangle\}$ and $\{|3\rangle,|4\rangle\}$ subspaces, as follows:

$$
U_{\mathrm{SE}}(\tau)=U_{\mathrm{SE}}^{12}(\tau) U_{\mathrm{SE}}^{34}(\tau)
$$

where the individual echo propagators are given by:

$$
\begin{aligned}
U_{\mathrm{SE}}^{12}(\tau) & =\exp \left\{-i \tau \omega^{12} \cdot I^{12}\right\} R_{z}^{12}(\pi) \exp \left\{-i \tau \omega^{12} \cdot I^{12}\right\} \\
U_{\mathrm{SE}}^{34}(\tau) & =i \Phi^{34}\left(\cos \left(\theta_{\mathrm{ST}}\right) \omega_{\mathrm{e}} \tau\right) R_{z}^{34}(\pi) .
\end{aligned}
$$

The group properties of $\mathrm{SU}(2)^{55}$ may be used to write the effective spin-echo propagator in subspace $\{|1\rangle,|2\rangle\}$ as follows:

$$
U_{\mathrm{SE}}^{12}(\tau)=\exp \left\{-i \xi^{12} \mathbf{n}^{12} \cdot I^{12}\right\}
$$

where the rotation axis $\mathbf{n}^{12}$ and rotation angle $\xi^{12}$ are given by the following expressions:

$$
\begin{aligned}
& \mathbf{n}^{12}=\csc \left(\frac{1}{2} \xi^{12}\right)\left[\begin{array}{c}
\sin \left(2 \theta_{\mathrm{ST}}\right) \sin ^{2}\left(\frac{1}{2} \tau \omega_{\mathrm{e}}\right) \\
0 \\
\cos ^{2}\left(\frac{1}{2} \tau \omega_{\mathrm{e}}\right)-\cos \left(2 \theta_{\mathrm{ST}}\right) \sin ^{2}\left(\frac{1}{2} \tau \omega_{\mathrm{e}}\right)
\end{array}\right], \\
& \xi^{12}=2 \cos ^{-1}\left(\cos \left(\theta_{\mathrm{ST}}\right) \sin \left(\tau_{1} \omega_{\mathrm{e}}\right)\right) .
\end{aligned}
$$

and the rotation axis is normalised such that $\mathbf{n}^{12} \cdot \mathbf{n}^{12}=1$.

\subsection{The M2S sequence}

The M2S pulse sequence is shown in Fig. 2. The sequence consists of 5 elements which operate as follows in the nearequivalence limit: ${ }^{37,38}$ (i) an initial $90_{y}$ pulse converts longitudinal magnetisation into transverse magnetisation, corresponding to single-quantum coherences within the triplet manifold; (ii) a set of $2 n$ consecutive spin echoes converts the single-quantum triplet-triplet coherences into coherences between the outer triplet states and the singlet state; (iii) a central $90_{x}$ pulse generates a zero-quantum coherence between the central triplet state and the singlet state; (iv) a delay interval adjusts the phase of the zero-quantum coherence; (v) a final echo train converts the zero-quantum coherence into a population difference between the central triplet state and the singlet state. If all these elements work perfectly, the theoretical limit of $\zeta_{\max }=\sqrt{2 / 3}$ is achieved for the magnetisation-to-singlet transformation amplitude (eqn (4)).

\section{a}

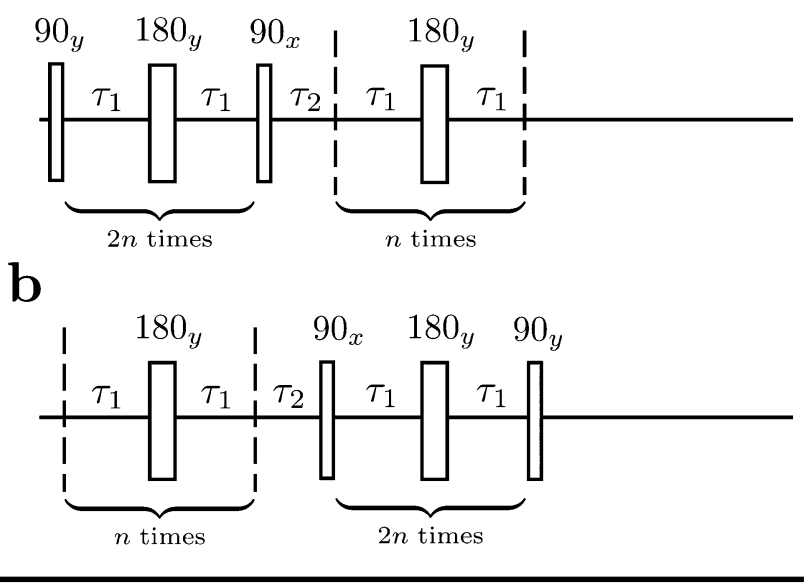

C

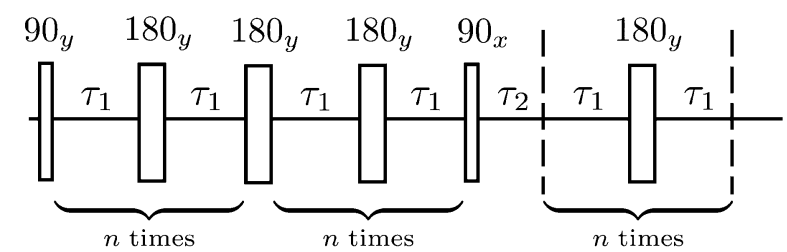

d

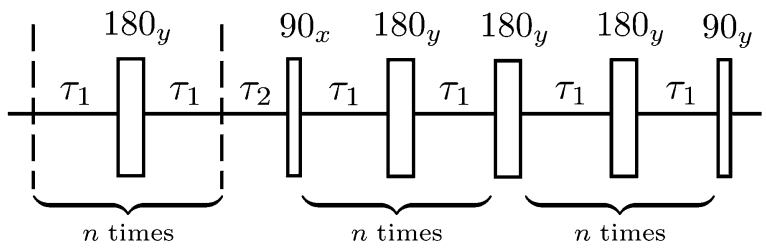

Fig. 2 (a) Magnetisation-to-Singlet (M2S) pulse sequence. (b) Singlet-toMagnetisation (S2M) pulse sequence. The S2M sequence is defined here as the chronological reverse of the M2S sequence, including the final pulse. (c) Generalised Magnetisation-to-Singlet (gM2S) pulse sequence. (d) Generalised Singlet-to-Magnetisation (gS2M) pulse sequence. The gS2M sequence is defined as the chronological reverse of the gM2S sequence, including the final pulse.

The S2M sequence is the chronological reverse of M2S (Fig. 4(b)). As defined here, M2S includes a final $90_{y}$ pulse and converts singlet order back into $z$-magnetisation. The overall amplitude for converting $z$-magnetisation into singlet order by M2S, and back again into $z$-magnetisation by $\mathrm{S} 2 \mathrm{M}$, is given by $\zeta_{\max }^{2}$, which has the maximum achievable value of $2 / 3$, for the case of unitary transformations.

As originally described, ${ }^{37}$ the M2S delays $\tau_{1}$ and $\tau_{2}$, and the loop number $n$, are specified as follows:

$$
\begin{aligned}
\tau_{1} & =\tau_{2}=(4 J)^{-1} \\
n & =\operatorname{round}(\pi J / 4 \Delta) .
\end{aligned}
$$

Tayler et $a l .{ }^{38}$ proposed modified timings, given by:

$$
\begin{aligned}
\tau_{1} & =\tau_{2}=\pi /\left(2 \omega_{\mathrm{e}}\right) \\
n & =\operatorname{round}\left(\pi / 2 \arctan \left(\theta_{\mathrm{ST}}\right)\right),
\end{aligned}
$$




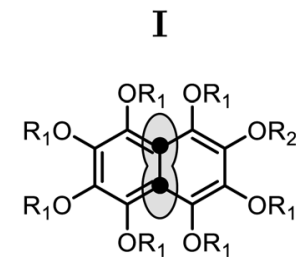

$$
\begin{aligned}
& \mathrm{R}_{1}=\mathrm{CD}_{3} \\
& \mathrm{R}_{2}=\mathrm{CD}\left(\mathrm{CD}_{3}\right)_{2}
\end{aligned}
$$$$
\mathrm{R}_{1}=\mathrm{C}\left(\mathrm{CD}_{3}\right)_{3}
$$$$
\mathrm{R}_{2}=\mathrm{CD}\left(\mathrm{CD}_{3}\right)_{2}
$$

Fig. 3 Chemical structures of compound (I) 1,2,3,4,5,6,8-heptakis(methoxy- $\left.d_{3}\right)$-7-((propan-2-yl- $\left.d_{7}\right)$ oxy)- $(4 a, 8 a)-{ }^{13} C_{2}$-naphthalene and compound (II) 1-(iso-propyl- $d_{7}$ )-4-(tert-butyl- $d_{9}$ )-(Z)-but-2-enedioate. The spin pair used for the singlet NMR experiments is indicated by a lasso. Side-chain protons have been replaced by deuterons as indicated by $R_{1}$ and $R_{2}$.

\section{a}

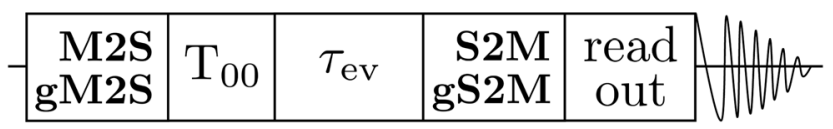

b

C

$\mathrm{T}_{00}$

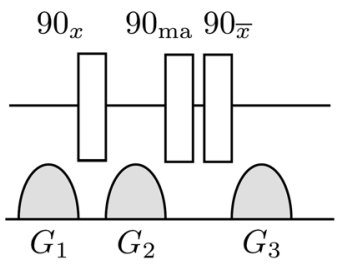

read out

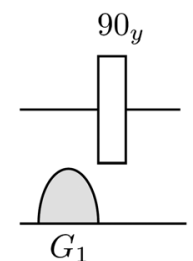

Fig. 4 (a) General singlet NMR pulse sequence consisting of an M2S/gM2S block, a singlet order filtration element $\left(T_{00}\right)$, an evolution interval $\tau_{\text {ev }}$ during which a continuous-wave (CW) rf field may be applied, an S2M/gS2M block, a read-out sequence for generating transverse magnetisation, and signal detection. (b) The singlet filter sequence consists of a set of radio-frequency pulses and field gradient pulses. The phase angle "ma" indicates the magic angle $\simeq 54.7^{\circ} .^{56}$ (c) The read out excitation sequence consists of a field gradient pulse for suppressing undesirable antiphase signal components, followed by excitation of transverse magnetisation by a $90_{y}$ pulse.

where $\omega_{e}$ is given by eqn (10). Eqn (21) and (22) converge to the same values for $\tau$ and $n$ in the near-equivalence limit $\left(\theta_{\mathrm{ST}} \approx 0\right)$.

The M2S sequence includes two spin echo trains, before and after the central $90_{x}$ pulse (see Fig. 2(a)). Ideally, these spin echo trains generate a rotation around the $x$-axis in the $\{|1\rangle,|2\rangle\}$ subspace, through the angles of $\pi$ (for the first spin echo train, consisting of $2 n$ echoes) and $\pi / 2$ (for the second spin echo train, consisting of $n$ echoes). In both cases the rotation axis is ideally given by

$$
\mathbf{n}^{12}=\left[\begin{array}{l}
1 \\
0 \\
0
\end{array}\right] \quad \text { (for M2S) }
$$

The parameter choices of eqn (21) and (22) both lead to the rotation axis in eqn $(23)$ in the near-equivalence limit $\left(\theta_{\mathrm{ST}} \approx 0\right)$. The same condition may be imposed for a wide range of $\theta_{\mathrm{ST}}$ values by choosing the echo delay $\tau_{1}$ to satisfy

$$
\tau_{1}^{*}=2 \omega_{\mathrm{e}}^{-1} \tan ^{-1}\left(1 / \sqrt{\cos \left(2 \theta_{\mathrm{ST}}\right)}\right),
$$

This leads to the following effective rotation angle for each spin echo

$$
\xi^{12}=2 \sec ^{-1}\left(\frac{\cos \left(\theta_{\mathrm{ST}}\right)}{\sqrt{\cos \left(2 \theta_{\mathrm{ST}}\right)}}\right) .
$$

In the limit of near equivalence the echo delay reduces to $\tau_{1}^{*} \approx 1 /(4 J)$ recovering the parameters in eqn (21).

The total rotation angle of the second spin echo train ideally satisfies the condition

$$
n \xi^{12}=\pi / 2,
$$

which implies that the total rotation angle of the first spin echo train (which is twice as long as the second) is equal to $\pi$, as required. Clearly, eqn (26) can only be satisfied when the angle $\xi^{12}$ happens to be an integer sub-multiple of $\pi / 2$. This is not always true. In the general case, the best one can do is to set the optimal echo number $n^{*}$ as follows

$$
n^{*}=\operatorname{round}\left(\pi /\left(2 \xi^{12}\right)\right)
$$

so that eqn (26) is approximately satisfied.

The two echo trains of the M2S sequence are separated by a $90 x$ pulse followed by a free evolution interval of duration $\tau_{2}$. This evolution interval allows the singlet and central triplet states to come into phase. ${ }^{38}$ The derivation of the optimal evolution delay $\tau_{2}{ }^{*}$ is straightforward but rather lengthy and is given in the ESI. $\dagger$ The result is

$$
\tau_{2}^{*}=\omega_{\mathrm{e}}{ }^{-1} \tan ^{-1}\left(\cos \left(\theta_{\mathrm{ST}}\right) \cot \left(2 n^{*} \tau_{1}^{*} \omega_{\mathrm{e}} \cos \left(\theta_{\mathrm{ST}}\right)\right)\right) .
$$

The optimal interval $\tau_{2}{ }^{*}$ for M2S reduces to $1 /(4 J)$ in the limit of near-equivalence, agreeing with eqn (21).

The orange curve in Fig. 1 shows the predicted performance of M2S as a function of the singlet-triplet mixing angle $\theta_{\mathrm{ST}}$, using the optimised M2S parameters summarized in Table 1. Simulations have also been performed for the literature solutions given in eqn (21) and eqn (22), and are not substantially different.

Fig. 1 shows that as $\theta_{\mathrm{ST}}$ increases from a low value, the performance of M2S oscillates in a saw-tooth fashion, with peaks at those values of mixing angles $\theta_{\mathrm{ST}}$ for which eqn (26) is satisfied exactly. Dips in performance are between these special values of $\theta_{\mathrm{ST}}$.

Eqn (24) does not admit any physical solutions at all for $\theta_{\text {ST }} \geq \pi / 4$. This indicates a fundamental limitation of the M2S approach. In reality, as shown in Fig. 1, the performance of M2S declines steeply, well before the absolute cutoff at $\theta_{\mathrm{ST}}=\pi / 4$.

\subsection{The gM2S sequence}

The gM2S sequence is shown in Fig. 2(c). It is very similar to the M2S sequence, but with the initial $2 n$-fold echo block split into two $n$-fold echo blocks separated by a single $180_{y}$ pulse. The optimal values of the delays $\tau_{1}{ }^{*}$ and $\tau_{2}{ }^{*}$ and the echo number $n^{*}$ 
are given in terms of the spin system parameters $\Delta$ and $J$ in Table 1.

For the M2S sequence, each spin echo element is designed to generate a rotation around the $x$-axis in the $\{|1\rangle,|2\rangle\}$ subspace (eqn (23)). For the gM2S sequence, on the other hand, each spin echo element $\left(\tau_{1}-180_{y}-\tau_{1}\right)$ is designed to generate a rotation around a tilted axis in the $\{|1\rangle,|2\rangle\}$ subspace of the form

$$
\mathbf{n}^{12}=\frac{1}{\sqrt{2}}\left[\begin{array}{l}
1 \\
0 \\
1
\end{array}\right] \quad \text { (for gM2S). }
$$

This implies that the rotation axis is in the $x z$-plane of the $\{|1\rangle,|2\rangle\}$ subspace, subtending an angle of $\pi / 4$ with the $x$ and $z$ axes.

Eqn (29) is satisfied by choosing the following value for the optimal echo delay $\tau_{1}^{*}$

$$
\begin{aligned}
\tau_{1}^{*} & =2 \omega_{\mathrm{e}}^{-1} \tan ^{-1}\left(1 / \sqrt{\cos \left(2 \theta_{\mathrm{ST}}\right)+\sin \left(2 \theta_{\mathrm{ST}}\right)}\right) \\
& =\omega_{\mathrm{e}}^{-1} \cos ^{-1}\left(\frac{1-\tan \left(\theta_{\mathrm{ST}}\right)}{1+\cot \left(\theta_{\mathrm{ST}}\right)}\right)
\end{aligned}
$$

where the effective rotation frequency $\omega_{\mathrm{e}}$ is defined in eqn (10). The optimal gM2S delay $\tau_{1}^{*}$ is well-defined as long as $(J+(1-\sqrt{2}) \Delta \geq 0)$. This condition allows physically realisable solutions for gM2S for a wide range of mixing angles $0<\theta_{\mathrm{ST}}<3 \pi / 8$. The upper limit of $\theta_{\mathrm{ST}}=3 \pi / 8=67.5^{\circ}$ is much larger than the M2S limit of $\theta_{\mathrm{ST}}=\pi / 4=45^{\circ}$.

The effective rotation angle in the $\{|1\rangle,|2\rangle\}$ subspace for the spin echo element $\left(\tau_{1}^{*}-180_{y}-\tau_{1}^{*}\right)$ is given by

$$
\xi^{12}=2 \sec ^{-1}\left(\frac{\cos \left(\theta_{\mathrm{ST}}\right)+\sin \left(\theta_{\mathrm{ST}}\right)}{\sqrt{\cos \left(2 \theta_{\mathrm{ST}}\right)+\sin \left(2 \theta_{\mathrm{ST}}\right)}}\right) .
$$

The rotation angle for $n$ repetitions of the spin echo element $\left(\tau_{1}^{*}-180_{y}-\tau_{1}^{*}\right)$ is equal to $n \xi^{12}$. In the case that $\xi^{12}$ is an integer submultiple of $\pi$, an optimal loop number $n^{*}$ may be found such that

$$
n^{*} \xi^{12}=\pi
$$

In the general case where $\xi^{12}$ is not an integer submultiple of $\pi$, the best solution for the loop number $n$ is given by

$$
n^{*}=\operatorname{round}\left(\pi / \xi^{12}\right) \text {. }
$$

If eqn (32) is satisfied exactly, the propagation operator in the $\{|1\rangle,|2\rangle\}$ subspace for a sequence of $n^{*}$ spin echoes is given by

$$
\begin{aligned}
U_{\mathrm{SE}}^{n^{*}}\left(\tau_{1}{ }^{*}\right) & =\exp \left\{-i \pi \mathbf{n}^{12} \cdot \mathbf{I}^{12}\right\} \\
& =R_{y}{ }^{12}(\pi / 4) R_{z}{ }^{12}(\pi) R_{y}{ }^{12}(-\pi / 4) \\
& =R_{y}{ }^{12}(\pi / 2) R_{z}{ }^{12}(\pi) .
\end{aligned}
$$

From eqn (16), the propagator in $\{|1\rangle,|2\rangle\}$ space for a single $180_{y}$ pulse is given by $R_{z}{ }^{12}(\pi)$. Providing that eqn (32) is satisfied exactly, the propagator for two $n^{*}$-fold gM2S echo trains, separated by a single $180_{y}$ pulse, is given by

$$
\begin{aligned}
& U_{\mathrm{SE}}^{n^{*}}\left(\tau_{1}{ }^{*}\right) R_{y}(\pi) U_{\mathrm{SE}}^{n^{*}}\left(\tau_{1}{ }^{*}\right)=R_{y}{ }^{12}(\pi / 2) R_{z}{ }^{12}(\pi) \cdot R_{z}{ }^{12}(\pi) \\
& \times R_{y}{ }^{12}(\pi / 2) R_{z}^{12}(\pi) \\
& =R_{y}{ }^{12}(\pi / 2) R_{z}{ }^{12}(3 \pi) R_{y}{ }^{12}(-\pi / 2) \\
& =-R_{y}{ }^{12}(\pi / 2) R_{z}^{12}(\pi) R_{y}{ }^{12}(-\pi / 2) \\
& =-R_{x}^{12}(\pi) \text {. }
\end{aligned}
$$

This implies that the sequence of two $n^{*}$-fold gM2S echo trains, separated by a single $180_{y}$ pulse, induces a $\pi$ rotation in $\{|1\rangle,|2\rangle\}$ space, which is the same result as the single echo train used in the M2S sequence. The advantage of the gM2S strategy is that solutions may be found for a much wider range of singlettriplet mixing angles $\theta_{\mathrm{ST}}$ than for the M2S.

The rest of the gM2S sequence operates in the same way as the M2S sequence. ${ }^{37,38}$ However, the correct choice of the $\tau_{2}$ delay requires some detailed analysis, which is presented in the ESI. $\dagger$ The optimal value of the $\tau_{2}$ delay is given by

$$
\tau_{2}{ }^{*}=\omega_{\mathrm{e}}{ }^{-1}\left|\tan ^{-1}\left(\cot \left(2 n^{*} \tau_{1}{ }^{*} \omega_{\mathrm{e}} \cos \left(\theta_{\mathrm{ST}}\right)\right) \sec \left(\theta_{\mathrm{ST}}\right)\right)\right|+2 \pi \omega_{\mathrm{e}}{ }^{-1} \delta_{1 n^{*}},
$$

where $\delta_{m n}$ is the Kronecker delta

$$
\delta_{m n}= \begin{cases}0 & \text { for } m \neq n \\ 1 & \text { for } m=n\end{cases}
$$

and $|x|$ represents the absolute value of $x$.

The blue curve in Fig. 1 shows the performance of the gM2S sequence as a function of $\theta_{\mathrm{ST}}$, with timing parameters specified in Table 1. The circles indicate singlet-triplet mixing angles at which eqn (32) is exactly satisfied for integer loop numbers $n^{*}$. The gM2S sequence achieves the theoretical maximum transformation amplitude of $\zeta_{\max }=\sqrt{2 / 3}$ at those points, which include the centre of the intermediate regime at $\theta_{\mathrm{ST}}=\pi / 4$. There are dips in performance between these special values of $\theta_{\mathrm{ST}}$, but the loss in amplitude is not severe. The gM2S sequence fills the gap between the M2S and Sarkar sequences by providing excitation efficiencies which are reasonably close to the theoretical maximum.

Since the gM2S sequence is based on spin echo sequences, it is very robust with respect to static field inhomogeneity, radiofrequency field inhomogeneity, and resonance offsets - especially when composite pulses are used. The performance of gM2S with respect to resonance offsets and rf field variations is explored in the ESI, $\dagger$ where it is contrasted with the ADAPT scheme. $^{50}$

\section{Experiments}

Singlet NMR experiments were performed on solutions of two different compounds, in order to compare the performance of the gM2S and M2S sequences. Compound $I$ is the ${ }^{13} \mathrm{C}_{2}$-labelled 
Table 2 NMR parameters for compounds I and II

\begin{tabular}{lll}
\hline Compound & I & II \\
\hline Nucleus & ${ }^{13} \mathrm{C}$ & ${ }^{1} \mathrm{H}$ \\
$J[\mathrm{~Hz}]$ & 54.10 & 12.30 \\
$\Delta \delta[\mathrm{ppm}]$ & 0.061 & 0.024 \\
$\theta_{\mathrm{ST}}$ & $6.47^{\circ}(@ 9.4 \mathrm{~T})$ & $38.0^{\circ}(@ 9.4 \mathrm{~T})$ \\
$T_{1}[\mathrm{~s}]$ & $17.43 \pm 0.20$ & $8.69 \pm 0.01$
\end{tabular}

naphthalene derivative shown in Fig. 3. This substance contains a near-equivalent ${ }^{13} \mathrm{C}$ spin pair which supports singlet order with an exceptional lifetime in low magnetic field. ${ }^{57}$ Compound II is an asymmetric tert-butyl propyl maleate diester containing a magnetically inequivalent pair of ${ }^{1} \mathrm{H}$ nuclei. Non participating side-chain protons are replaced by deuterons to reduce dipole-dipole relaxation contributions (see Fig. 3). ${ }^{3,5}$ The NMR parameters for the spin pairs in both compounds are summarised in Table 2.

The synthesis of compound I is described in ref. 58. The experiments used a $0.1 \mathrm{M}$ solution of compound $\mathbf{I}$ in deuterated acetone, contained in a $5 \mathrm{~mm}$ Wilmad LPV tube with the sample volume limited to $0.35 \mathrm{ml}$. The sample was degassed by several freeze-thaw-cycles. The synthesis of compound II is
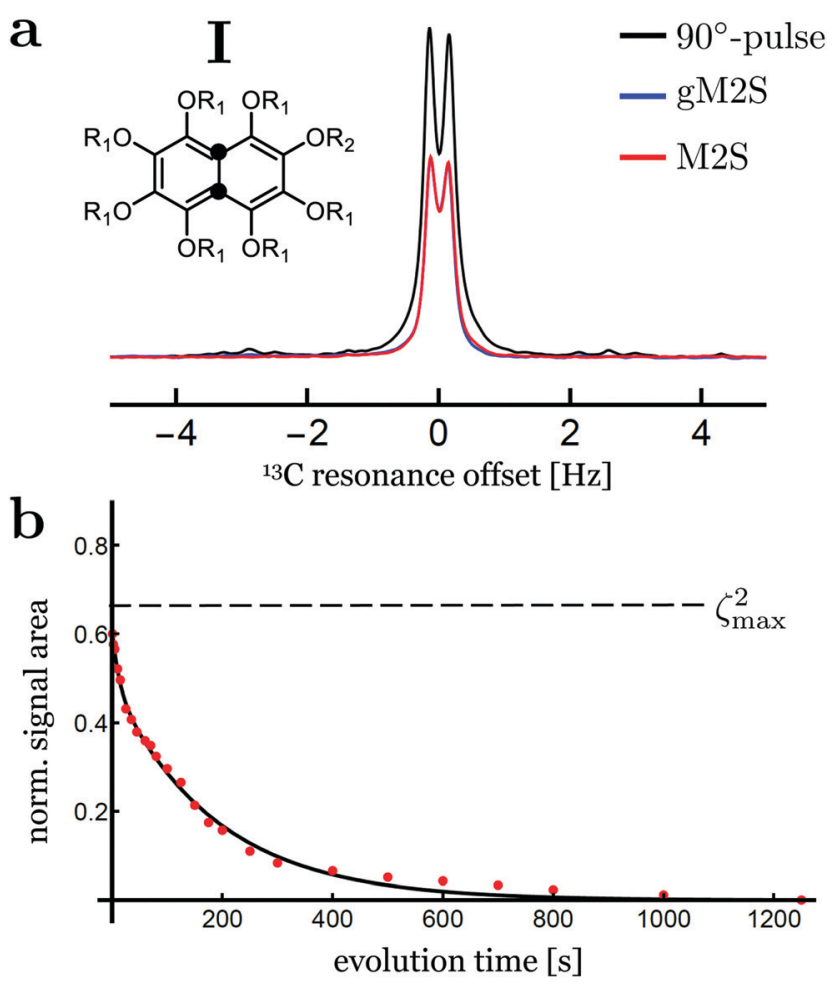

Fig. 5 (a) Singlet-filtered ${ }^{13} \mathrm{C}$ NMR spectra for compound I, using the M2S and gM2S sequences. A single pulse-acquire spectrum is shown for comparison. The spectra for M2S and gM2S are almost superimposed. (b) Singlet order decay as a function of the evolution interval $\tau_{\mathrm{ev}}$, applying a spin-locking field with nutation frequency $\sim 2 \mathrm{kHz}$ during the evolution interval. The plotted signal amplitude is normalised against the singlepulse-acquire spectrum. The solid line shows the best fit to a biexponential decay. The theoretical maximum of $\zeta_{\max }^{2}=2 / 3$ is indicated by the horizontal dashed line.
Table 3 M2S and gM2S parameters used for compound $\mathbf{I}$. The values of $\tau_{1}{ }^{*}$ and $\tau_{2}{ }^{*}$ are given by the analytical solutions in Table 1 . The experimental delays after empirical optimisation are given by $\tau_{1}^{\exp }$ and $\tau_{2}^{\exp }$. The radiofrequency field amplitude for the strong $\mathrm{rf}$ pulses is given as a nutation frequency $\omega_{\text {nut }} / 2 \pi$

\begin{tabular}{lcc}
\hline & M2S & gM2S \\
\hline$n^{*}=n^{\exp }$ & 7 & 11 \\
$\tau_{1}^{*}[\mathrm{~ms}]$ & 4.64 & 4.33 \\
$\tau_{1}^{\exp }[\mathrm{ms}]$ & 4.63 & 4.33 \\
$\tau_{2}^{*}[\mathrm{~ms}]$ & 4.48 & 1.86 \\
$\tau_{2}^{\exp }[\mathrm{ms}]$ & 4.63 & 2.37 \\
$\omega_{\text {nut }} / 2 \pi[\mathrm{kHz}]$ & 30.0 & 30.0 \\
\hline
\end{tabular}

described in ref. 48. The experiments were performed on a degassed $1.7 \mathrm{mM}$ solution in deuterated chloroform, with the sample volume restricted to $0.3 \mathrm{ml}$ within a $5 \mathrm{~mm}$ Shigemi LPV tube to limit convection effects. The degassing procedure also consisted of several freeze-thaw-cycles.

All spectra were acquired at a magnetic field of $9.4 \mathrm{~T}$. All pulses in Fig. 2 were replaced by their composite pulse counterparts to compensate for possible static and radio-frequency field inhomogeneities. ${ }^{59,60}$ Each $180_{y}$ pulse was replaced by a composite inversion pulse of the form $90_{x} 180_{y} 90_{x}{ }^{59}$ All $90_{\phi}$ pulses were replaced by the constant-rotation composite pulse $180_{97.2+\phi} 360_{291.5+\phi} 180_{97.2+\phi} 90_{\phi}$ given in ref. 60. Individual data sets employed a basic two-step phase cycle and were averaged over two transients before post-processing using $0.25 \mathrm{~Hz}$ line broadening.

The general procedure for the singlet NMR experiments is shown in Fig. 4(a). Singlet order is generated using either a M2S or a gM2S sequence. This is followed by a singlet filtration step, denoted $\mathrm{T}_{00}$, which is implemented by a sequence of radiofrequency pulses and field gradients. This suppresses all signals not passing through singlet order. ${ }^{56}$ For singlet lifetime measurements an additional evolution interval $\tau_{\mathrm{ev}}$ is inserted, which may include the application of a spin-locking field. The singlet order is reconverted into $z$-magnetisation by applying a $\mathrm{S} 2 \mathrm{M}$ or a gS2M pulse sequence (including the final $90_{y}$ pulse, see Fig. 2(b and d)). The $z$-magnetisation is allowed to rest for a further delay which may include another field gradient pulse. This implements a $z$-filter which cleans up the final signal by removing undesirable signal components. A final $90_{y}$ pulse induces transverse magnetisation and the NMR signal is detected.

The gM2S and M2S parameters were set by fixing the echo numbers to the values specified in Table 1 and optimising the delays $\tau_{1}$ and $\tau_{2}$ empirically in a small interval centred around the analytic solutions.

\section{Results and discussion}

\subsection{Compound I}

Singlet-filtered NMR signals for compound I are shown in Fig. 5(a), using the optimised M2S/S2M and gM2S/gS2M sequences. A simple pulse-acquire spectrum is also shown for reference. 
The pulse sequence parameters for the M2S and gM2S sequences are summarised in Table 3.

The M2S and gM2S sequences display very similar performance, as expected for the near-equivalence regime. Integration of the resulting spectra and comparison with the pulse-acquire reference indicates that both sequences pass approximately $60 \%$ of the initial magnetisation through singlet order and back to magnetisation ( $\zeta^{2} \simeq 60 \%$ for M2S and 59\% for gM2S). This is respectably close to the theoretical maximum of $\zeta_{\max }^{2}=2 / 3 \simeq 66.7 \%$. The remaining loss may be attributed to relaxation during the pulse sequences and residual pulse imperfections.

Fig. 5(b) shows the decay of singlet order for compound I using the gM2S/gS2M sequence with $2 \mathrm{kHz}$ continuous-wave irradiation during the evolution interval. The decay curve displays a bi-exponential behaviour of the form: $s\left(\tau_{\mathrm{ev}}\right)=A_{1} \exp \left(-\tau_{\mathrm{ev}} / T_{1}\right)+$ $A_{\mathrm{S}} \exp \left(-\tau_{\mathrm{ev}} / T_{\mathrm{S}}\right)$ with fit parameters $A_{1}=0.12 \pm 1.6, A_{\mathrm{S}}=0.49 \pm 0.02$, $T_{1}=12.2 \pm 4.1 \mathrm{~s}$ and $T_{\mathrm{S}}=186 \pm 9 \mathrm{~s}$. The major component may be identified as long-lived singlet order with a relaxation time constant of $T_{\mathrm{S}} \simeq 186 \mathrm{~s}$, which is approximately 10 times longer than the longitudinal relaxation time constant $T_{1}=17.4 \pm 0.2 \mathrm{~s}$. Comparable results have been reported previously at this magnetic field, albeit using the M2S sequence instead of the gM2S sequence. $^{57}$

At this stage the bi-exponential decay behaviour of singlet order in compound $\mathbf{I}$ is not fully understood. A possible explanation may involve the weak scalar couplings to nearby deuterons. These are known to induce scalar relaxation of the second kind (SR2K) resulting in a non-mono-exponential decay. ${ }^{61}$ Since the sample volume of compound I was not restricted, convection effects could also contribute to the bi-exponential decay behaviour. ${ }^{62}$

\subsection{Compound II}

The spectra for compound II after singlet order excitation via M2S and gM2S are shown in Fig. 6(a). The experimentally optimised M2S and gM2S parameters are given in Table 4.

The proton spin system of compound II has a singlet-triplet mixing angle of $\theta_{\mathrm{ST}}=38.0^{\circ}$, which places it firmly in the intermediate-coupling regime. In this case a large differences in performance is observed for the M2S and gM2S sequences.

The signal amplitude observed for the M2S sequence is weak for this system, with an integrated amplitude of only $\sim 19 \%$ of the pulse-acquire spectrum.

The gM2S sequence gives a much stronger singlet-filtered NMR signal. The integrated amplitude of the singlet-filtered NMR spectrum is $\sim 50 \%$ of the pulse-acquire spectrum, which is a respectable fraction of the theoretical maximum, $\zeta_{\max }^{2}=$ $2 / 3 \simeq 66.7 \%$.

The decay of singlet order for compound II under $2 \mathrm{kHz}$ continuous wave irradiation is shown in Fig. 6(b). The decay curve is well approximated by a mono-exponential decay of the form: $s\left(\tau_{\mathrm{ev}}\right)=A_{\mathrm{S}} \exp \left(-\tau_{\mathrm{ev}} / T_{\mathrm{S}}\right)$ with fit parameters $A_{\mathrm{S}}=0.52 \pm 0.02$ and $T_{\mathrm{S}}=77.6 \pm 1.0 \mathrm{~s}$. The singlet order decay constant for compound II is therefore approximately ten times longer than the time constant for thermalisation of longitudinal magnetisation, $T_{1}=8.69 \pm 0.01 \mathrm{~s}$.
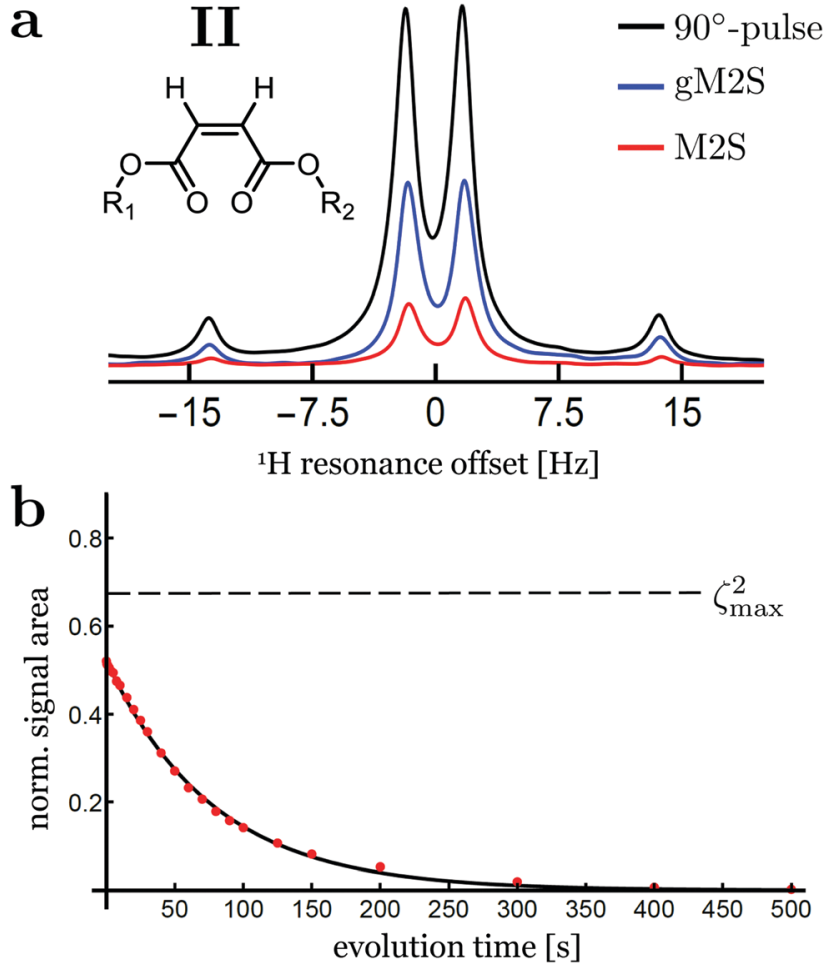

Fig. 6 (a) Singlet-filtered ${ }^{1} \mathrm{H}$ NMR spectra for compound II, using the M2S and gM2S sequences. A single pulse-acquire spectrum is shown for comparison. (b) Singlet order decay as a function of the evolution interval $\tau_{\mathrm{ev}}$, applying a spin-locking field with nutation frequency $\sim 2 \mathrm{kHz}$ during the evolution interval. The plotted signal amplitudes are normalised against the single-pulse-acquire spectrum. The solid line shows the best fit to an exponential decay. The theoretical maximum of $\zeta_{\max }^{2}=2 / 3$ is indicated by the horizontal dashed line.

Table 4 M2S and gM2S parameters used for compound II. The values of $\tau_{1}{ }^{*}$ and $\tau_{2}{ }^{*}$ are given by the analytical solutions in Table 1 . The experimental delays after empirical optimisation are given by $\tau_{1}^{\exp }$ and $\tau_{2}^{\exp }$. The radiofrequency field amplitude for the strong rf pulses is given as a nutation frequency $\omega_{\text {nut }} / 2 \pi$

\begin{tabular}{lcc}
\hline & M2S & gM2S \\
\hline$n^{*}=n^{\exp }$ & 1 & 2 \\
$\tau_{1}^{*}[\mathrm{~ms}]$ & 23.46 & 14.30 \\
$\tau_{1}^{\exp }[\mathrm{ms}]$ & 21.80 & 15.40 \\
$\tau_{2}^{*}[\mathrm{~ms}]$ & 11.16 & 1.20 \\
$\tau_{2}^{\exp }[\mathrm{ms}]$ & 12.50 & 1.24 \\
$\omega_{\text {nut }} / 2 \pi[\mathrm{kHz}]$ & 25.0 & 25.0 \\
\hline
\end{tabular}

\section{Conclusions}

To summarise, we have described a generalisation of the singlet-to-magnetisation (M2S) sequence. The proposed generalised-M2S sequence (gM2S) performs near-optimal singlet order excitation for spin-pair systems ranging from the nearequivalence limit, through the intermediate regime, to the boundary of strong inequivalence. We have given analytical solutions for the delays and loop numbers in the short-pulse limit. Small adjustments for finite pulse durations are readily implemented by empirical optimisation on the spectrometer. 
The performance of M2S and gM2S was evaluated experimentally in two model systems, containing spin-1/2 pairs in the near-equivalence and intermediate coupling regimes. In the near-equivalence regime, both $\mathrm{M} 2 \mathrm{~S}$ and gM2S achieve nearoptimal efficiency for the passage of transverse magnetisation through singlet order and back to transverse magnetisation. In the intermediate coupling regime, on the other hand, the gM2S sequence greatly outperforms the M2S sequence.

Although the implementation of gM2S is somewhat more complex than M2S, the gM2S overcomes the restriction of the M2S to near-equivalent systems and enables the study of new molecular systems. ${ }^{37,38}$ We therefore anticipate its incorporation into a wide class of singlet-assisted NMR experiments. ${ }^{15-17,25-28,31-34}$

Further extensions to the case of heteronuclear spin systems are feasible, for example in the context of parahydrogenenhanced NMR. ${ }^{63-65}$

\section{Conflicts of interest}

There are no conflicts to declare.

\section{Acknowledgements}

This research was supported by the European Research Council (786707-FunMagResBeacons) and the Engineering and Physical Sciences Research Council (EPSRC-UK), grant numbers EP/ P009980/1 and EP/P030491/1. A. J. acknowledges funding from the National Science Foundation under award \#CHE 1710046 and a Diamond Jubilee Visiting Fellowship from the University of Southampton.

\section{References}

1 M. Carravetta, O. G. Johannessen and M. H. Levitt, Phys. Rev. Lett., 2004, 92, 153003.

2 M. Carravetta and M. H. Levitt, J. Am. Chem. Soc., 2004, 126, 6228-6229.

3 M. Carravetta and M. H. Levitt, J. Chem. Phys., 2005, 122, 214505.

4 M. H. Levitt, Annu. Rev. Phys. Chem., 2012, 63, 89-105.

5 G. Pileio and M. H. Levitt, J. Chem. Phys., 2009, 130, 214501.

6 G. Pileio, M. Carravetta, E. Hughes and M. H. Levitt, J. Am. Chem. Soc., 2008, 130, 12582-12583.

7 G. Stevanato, J. T. Hill-Cousins, P. Håkansson, S. S. Roy, L. J. Brown, R. C. D. Brown, G. Pileio and M. H. Levitt, Angew. Chem., Int. Ed., 2015, 54, 3740-3743.

8 T. Theis, G. X. Ortiz, A. W. J. Logan, K. E. Claytor, Y. Feng, W. P. Huhn, V. Blum, S. J. Malcolmson, E. Y. Chekmenev, Q. Wang and W. S. Warren, Sci. Adv., 2016, 2, e1501438.

9 S. J. Elliott, P. Kadeřávek, L. J. Brown, M. Sabba, S. Glöggler, D. J. O’Leary, R. C. D. Brown, F. Ferrage and M. H. Levitt, Mol. Phys., 2019, 117, 861-867.

10 M. H. Levitt, J. Magn. Reson., 2019, 306, 69-74.

11 N. Salvi, Annual Reports on NMR Spectroscopy, Academic Press, 2019, vol. 96, pp. 1-33.
12 J.-N. Dumez, Mol. Phys., 2019, 1-11.

13 S. Cavadini, J. Dittmer, S. Antonijevic and G. Bodenhausen, J. Am. Chem. Soc., 2005, 127, 15744-15748.

14 G. Pileio, J.-N. Dumez, I.-A. Pop, J. T. Hill-Cousins and R. C. D. Brown, J. Magn. Reson., 2015, 252, 130-134.

15 S. Cavadini and P. R. Vasos, Concepts Magn. Reson., Part A, 2008, 32, 68-78.

16 G. Pileio and S. Ostrowska, J. Magn. Reson., 2017, 285, 1-7.

17 M. C. Tourell, I.-A. Pop, L. J. Brown, R. C. D. Brown and G. Pileio, Phys. Chem. Chem. Phys., 2018, 20, 13705-13713.

18 L. Buljubasich, M. B. Franzoni, H. W. Spiess and K. Münnemann, J. Magn. Reson., 2012, 219, 33-40.

19 T. Theis, M. Truong, A. M. Coffey, E. Y. Chekmenev and W. S. Warren, J. Magn. Reson., 2014, 248, 23-26.

20 Y. Zhang, P. C. Soon, A. Jerschow and J. W. Canary, Angew. Chem., Int. Ed., 2014, 53, 3396-3399.

21 Y. Zhang, K. Basu, J. W. Canary and A. Jerschow, Phys. Chem. Chem. Phys., 2015, 17, 24370-24375.

22 S. S. Roy, P. J. Rayner, P. Norcott, G. G. R. Green and S. B. Duckett, Phys. Chem. Chem. Phys., 2016, 18, 24905-24911.

23 S. S. Roy, P. Norcott, P. J. Rayner, G. G. R. Green and S. B. Duckett, Chem. - Eur. J., 2017, 23, 10496-10500.

24 S. S. Roy, G. Stevanato, P. J. Rayner and S. B. Duckett, J. Magn. Reson., 2017, 285, 55-60.

25 K. F. Sheberstov, H.-M. Vieth, H. Zimmermann, B. A. Rodin, K. L. Ivanov, A. S. Kiryutin and A. V. Yurkovskaya, Sci. Rep., 2019, 9, 1-11.

26 A. S. Kiryutin, B. A. Rodin, A. V. Yurkovskaya, K. L. Ivanov, D. Kurzbach, S. Jannin, D. Guarin, D. Abergel and G. Bodenhausen, Phys. Chem. Chem. Phys., 2019, 21, 13696-13705.

27 W. Iali, S. S. Roy, B. J. Tickner, F. Ahwal, A. J. Kennerley and S. B. Duckett, Angew. Chem., 2019, 131, 10377-10381.

28 S. S. Roy, P. J. Rayner, M. J. Burns and S. B. Duckett, J. Chem. Phys., 2020, 152, 014201.

29 N. Salvi, R. Buratto, A. Bornet, S. Ulzega, I. Rentero Rebollo, A. Angelini, C. Heinis and G. Bodenhausen, J. Am. Chem. Soc., 2012, 134, 11076-11079.

30 R. Buratto, D. Mammoli, E. Chiarparin, G. Williams and G. Bodenhausen, Angew. Chem., Int. Ed., 2014, 53, 11376-11380.

31 W. Iali, P. J. Rayner and S. B. Duckett, Sci. Adv., 2018, 4, eaao6250.

32 A. Sadet, C. Stavarache, F. Teleanu and P. R. Vasos, Sci. Rep., 2019, 9, 1-7.

33 S. Yang, J. McCormick, S. Mamone, L.-S. Bouchard and S. Glöggler, Angew. Chem., Int. Ed., 2019, 58, 2879-2883.

34 T. Kress, A. Walrant, G. Bodenhausen and D. Kurzbach, J. Phys. Chem. Lett., 2019, 10, 1523-1529.

35 G. Pileio, Prog. Nucl. Magn. Reson. Spectrosc., 2010, 56, 217-231.

36 R. Sarkar, P. Ahuja, D. Moskau, P. R. Vasos and G. Bodenhausen, ChemPhysChem, 2007, 8, 2652-2656.

37 G. Pileio, M. Carravetta and M. H. Levitt, Proc. Natl. Acad. Sci. U. S. A., 2010, 107, 17135-17139.

38 M. C. D. Tayler and M. H. Levitt, Phys. Chem. Chem. Phys., 2011, 13, 5556-5560. 
39 R. Sarkar, P. Ahuja, P. R. Vasos and G. Bodenhausen, Phys. Rev. Lett., 2010, 104, 053001.

40 S. J. DeVience, R. L. Walsworth and M. S. Rosen, Phys. Rev. Lett., 2013, 111, 173002.

41 A. N. Pravdivtsev, A. S. Kiryutin, A. V. Yurkovskaya, H.M. Vieth and K. L. Ivanov, J. Magn. Reson., 2016, 273, 56-64.

42 B. A. Rodin, K. F. Sheberstov, A. S. Kiryutin, J. T. Hill-Cousins, L. J. Brown, R. C. D. Brown, B. Jamain, H. Zimmermann, R. Z. Sagdeev, A. V. Yurkovskaya and K. L. Ivanov, J. Chem. Phys., 2019, 150, 064201.

43 J. Jeener, Advances in Magnetic and Optical Resonance, Academic Press, 1982, vol. 10, pp. 1-51.

44 O. W. Sørensen, J. Magn. Reson., 1990, 86, 435-440.

45 M. H. Levitt, J. Magn. Reson., 2016, 262, 91-99.

46 R. Sarkar, P. R. Vasos and G. Bodenhausen, J. Am. Chem. Soc., 2007, 129, 328-334.

47 G. A. Morris, N. P. Jerome and L.-Y. Lian, Angew. Chem., Int. Ed., 2003, 42, 823-825.

48 B. Kharkov, X. Duan, E. S. Tovar, J. W. Canary and A. Jerschow, Phys. Chem. Chem. Phys., 2019, 21, 2595-2600. 49 G. Stevanato, J. Magn. Reson., 2017, 274, 148-162.

50 S. J. Elliott and G. Stevanato, J. Magn. Reson., 2019, 301, 49-55. 51 C. Laustsen, S. Bowen, M. S. Vinding, N. C. Nielsen and J. H. Ardenkjaer-Larsen, Magn. Reson. Med., 2014, 71, 921-926.

52 M. H. Levitt, Spin dynamics: basics of nuclear magnetic resonance, Wiley, 2001.

53 S. Vega and A. Pines, J. Chem. Phys., 1977, 66, 5624-5644.
54 S. Vega, J. Chem. Phys., 1978, 68, 5518-5527.

55 S. Scherer, Symmetrien und Gruppen in der Teilchenphysik, Springer Spektrum, 2016.

56 M. C. D. Tayler and M. H. Levitt, J. Am. Chem. Soc., 2013, 135, 2120-2123.

57 G. Stevanato, S. S. Roy, J. Hill-Cousins, I. Kuprov, L. J. Brown, R. C. D. Brown, G. Pileio and M. H. Levitt, Phys. Chem. Chem. Phys., 2015, 17, 5913-5922.

58 J. T. Hill-Cousins, I.-A. Pop, G. Pileio, G. Stevanato, P. HÅkansson, S. S. Roy, M. H. Levitt, L. J. Brown and R. C. D. Brown, Org. Lett., 2015, 17, 2150-2153.

59 M. H. Levitt and R. Freeman, J. Magn. Reson., 1979, 33, 473-476.

60 S. Wimperis, J. Magn. Reson., Ser. A, 1994, 109, 221-231.

61 S. J. Elliott, C. Bengs, L. J. Brown, J. T. Hill-Cousins, D. J. O'Leary, G. Pileio and M. H. Levitt, J. Chem. Phys., 2019, 150, 064315.

62 B. Kharkov, X. Duan, J. W. Canary and A. Jerschow, J. Magn. Reson., 2017, 284, 1-7.

63 S. Kadlecek, K. Emami, M. Ishii and R. Rizi, J. Magn. Reson., 2010, 205, 9-13.

64 J. Eills, G. Stevanato, C. Bengs, S. Glöggler, S. J. Elliott, J. Alonso-Valdesueiro, G. Pileio and M. H. Levitt, J. Magn. Reson., 2017, 274, 163-172.

65 J. Eills, J. Blanchard, T. Wu, C. Bengs, J. Hollenbach, D. Budker and M. Levitt, Polarization Transfer via Field Sweeping in Parahydrogen-Enhanced Nuclear Magnetic Resonance, ChemRxiv, 2019. 